\title{
Preparation and Properties of Carrageenan-g-Poly(Acrylic Acid)/Bentonite Superabsorbent Composite
}

\author{
Hossein Hosseinzadeh $^{1 *}$, Mina Sadeghzadeh², Mirzaagha Babazadeh ${ }^{2}$ \\ ${ }^{1}$ Chemistry Department, Payame Noor University, Tehran, Iran; ${ }^{2}$ Department of Chemistry, Science Faculty, Islamic Azad Univer- \\ sity, Tabriz Branch, Tabriz, Iran. \\ Email: ${ }^{*} h \_$hoseinzadeh@pnu.ac.ir
}

Received December $3^{\text {rd }}, 2010$; revised March 20 ${ }^{\text {th }} 2011$; accepted April 21 ${ }^{\text {st }}, 2011$.

\begin{abstract}
A novel biopolymer-based superabsorbent hydrogel composite based on kappa-carrageenan $(\kappa C)$ have been prepared via graft copolymerization of acrylic acid $(A A)$ in the presence of bentonite powder using methylenebisacrylamide $(M B A)$ as a crosslinking agent and ammonium persulfate (APS) as an initiator. The hydrogel structure was confirmed using FTIR spectroscopy and the morphology of the samples was examined by scanning electron microscopy (SEM). The affecting variables onto graft polymerization (i.e. AA, MBA and APS concentration, as well as the bentonite amount) were systematically optimized to achieve a hydrogel with swelling capacity as high as possible. The results of Brunauer-Emmett-Teller (BET) analysis showed that the average pore diameter of the synthesized hydrogel was $11.5 \mathrm{~nm}$. The effect of various salt media and solutions with different pHs on the swelling of the superabsorbent was also studied.
\end{abstract}

Keywords: Carrageenan, Bentonite, Poly(Acrylic Acid), Hydrogel, Composite

\section{Introduction}

Polymer hydrogels, as a sort of unique soft materials, have attracted more and more attention because of their academic importance and special applications [1,2]. Superabsorbent polymers (SAPs) are special soft and pliable polymeric materials that can be absorb large quantities of water, saline or physiological solutions while the absorbed solutions are not removable even under pressure $[3,4]$. Because of their superior properties, they have found extensive applications such as disposable diapers, feminine napkins, drug delivery systems, and soil for agriculture and horticulture [5-7].

For the majority of applications, the hydrogels have to possess high absorption capacity and elevated swelling rate and show a strong swollen gel. Hydrogels with high mechanical strength are required in some applications such as artificial cartilage [8,9], controlled drug delivery [10], hygiene and agricultural uses [1]. The gel strength can be improved through different methods such as surface crosslinking [1,11-13] and creating composite [1416] and nanocomposite structures [17-20].

Because of their exceptional properties, i.e. biocompatibility, biodegradability, renewability, and non-toxicity, polysaccharides are the main part of the natural-based superabsorbent hydrogels [21-24]. The higher production cost and low gel strength of these superabsorbents, however, restrict their application widely. To improve these limitations, inorganic compounds with low cost can be used. The introduction of inorganic fillers to a polymer matrix increases its strength and stiffness properties. Among inorganic compounds, special attention has been paid to clay minerals in the field of nanocomposites because of their small particle size and intercalation properties. Mineral powders are hydrated layered aluminosilicate with reactive $-\mathrm{OH}$ groups on the surface. The interaction of mineral powders, reactive site of natural polymers and monomers results in a superabsorbent composite. Superabsorbent composites based on synthetic polymers $[15,25]$ or natural polymers $[26,27]$ have been reported.

Carrageenan is a collective term for linear sulfated polysaccharides that are obtained commercially by alkaline extraction of certain species of red seaweeds [28]. Schematic diagram of the idealized structure of the repeat units for the most well-known and most important type of carrageenan family, kappa-carrageenan $(\kappa \mathrm{C})$, are 
presented in Figure 1. The presence of hydrophilic sulfate groups with high ionization tendency and less sensitivity to salt solution was our main idea for synthesis of carrageenan-based superabsorbent hydrogels.

Moreover high viscosity of carrageenan solutions decreased diffusion of molecular oxygen in the reaction mixture that consequently decreased the inhibiting effect of the oxygen in free radical polymerization process. This fact has already been observed in the case of other polysaccharides $[29,30]$. Therefore we were able to apply crosslinking graft copolymerization reaction under atmospheric conditions which causes brevity and simplicity in industrial processes.

In this work, we attempt to synthesize and characterize new superabsorbent composites based on kappa-carrageenan $(\kappa \mathrm{C})$ in the presence of bentonite particles. The preparation of the biopolymer-based superabsorbent composites can also improve the mechanical properties of materials and can lower the cost of the finished product compared with the synthetic counterparts as well as providing biodegradable characteristics. The reaction variables affecting the water absorbency of the $\kappa \mathrm{C}-\mathrm{g}$ poly(acrylic acid)/bentonite as well as the salt- and $\mathrm{pH}$ sensitivity of the hydrogels were investigated.

\section{Experimental}

The polysaccharide, $\kappa \mathrm{C}$ (chemical grade, MW 50000, from Condinson Co., Denmark) was used without further purification. Acrylic acid (from Merck) was used after vacuum distillation. MBA (from Fluka), APS (from Merck) and bentonite (from Aldrich, particle size $<5 \mu \mathrm{m}$ ) were used as received. All other chemicals were of analytical grade.

\subsection{Superabsorbent Composite Synthesis}

A pre-weighed amount of of $\kappa \mathrm{C}(0.30-0.70 \mathrm{~g})$ was added to $40 \mathrm{~mL}$ degassed distilled water in a 1-L reactor equipped with a mechanical stirrer (RZR 2021, a threeblade propeller type, Heidolph, Schwabach, Germany) and stirred for $10 \mathrm{~min}$. The reactor was placed in a thermostated water bath to control the reaction temperature at $80^{\circ} \mathrm{C}$. After complete dissolution of $\kappa \mathrm{C}$, various amounts of bentonite powder $(0.70-0.30 \mathrm{~g})$ were added to the $\kappa \mathrm{C}$ solution and allowed to stir for $10 \mathrm{~min}$. Then, APS initiator $(0.05-0.50 \mathrm{~g}$, dissolved in $5 \mathrm{~mL}$ water) was added to the reaction mixture and the mixture was stirred for 10 min. MBA (0.05 - $0.25 \mathrm{~g}$, dissolved in $5 \mathrm{~mL}$ water) and AA $(1.0-5.0 \mathrm{~g}$, completely neutralized with $\mathrm{NaOH})$ were poured into the reactor. All of the reactions were carried out at $80^{\circ} \mathrm{C}$ under an argon gas atmosphere. At the end of the propagation reaction, the gelly product was poured in ethanol $(300 \mathrm{ml})$ and allowed to dewater for 24 h. Then, the product was filtered and washed with 100

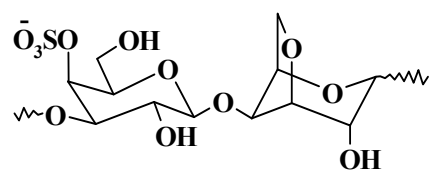

Figure 1. Repeating disaccharide units of kappa-carrageenan (кC).

$\mathrm{mL}$ ethanol. The filtered product was dried in an oven at $50^{\circ} \mathrm{C}$ for $10 \mathrm{~h}$. After grinding, the powdered superabsorbent composite was stored away from moisture, heat and light.

\subsection{Swelling Measurements}

An accurately weighed sample $(0.2 \pm 0.001 \mathrm{~g})$ of the powdered superabsorbent with average particle sizes between $40-60$ mesh $(250-350 \mu \mathrm{m})$ was immersed in distilled water $(200 \mathrm{~mL})$ and allowed to soak for $3 \mathrm{~h}$ at room temperature. The equilibrium swelling (ES) capacity was measured twice at room temperature according to a conventional tea bag (i.e. a 100 mesh nylon screen) method and using the following formula:

$$
(g / g)=\frac{\text { Weight of swollen gel }- \text { Weight of dried gel }}{\text { Weight of dried gel }}(1)
$$

\section{Results and Discussion}

\subsection{Synthesis and Characterization}

The hydrogel composite was prepared by graft copolymerization of acrylic acid onto $\kappa \mathrm{C}$ in the presence of a crosslinking agent and powdery bentonite (Scheme 1). Ammonium persulfate was used as an initiator. The persulfate is decomposed under heating and produced sulfate anion-radicals that abstract hydrogen from $-\mathrm{OH}$ groups of $\kappa \mathrm{C}$ backbones. So, this persulfate-saccharide redox system results in active centers capable to radically initiate polymerization of acrylic acid led to a graft copolymer. Since a crosslinking agent, e.g. MBA, is presented in the system, the copolymer comprises a crosslinked structure.

FTIR spectroscopy was used for identification of the hydrogel. In Figure 2, (a) represents the spectrum of the physical mixture of $\kappa \mathrm{C}$ and bentonite. In the layer silicate structure of bentonite, the hydroxyl groups show absorption bands at $3630-3680 \mathrm{~cm}^{-1}$. The broad band at $3200-$ $3400 \mathrm{~cm}^{-1}$ is due to stretching of $-\mathrm{OH}$ groups of the polysaccharide. In the spectrum of the composite ((b) in Figure 2), two new absorption peaks at 1579 and $1722 \mathrm{~cm}^{-1}$ are appeared. The characteristic band at $1572 \mathrm{~cm}^{-1}$ is due to $\mathrm{C}=\mathrm{O}$ asymmetric stretching in carboxylate anion that is reconfirmed by another peak at $1410 \mathrm{~cm}^{-1}$ which is related to the symmetric stretching mode of the carboxylate groups. The absorption band at $1722 \mathrm{~cm}^{-1}$ can be corres- 

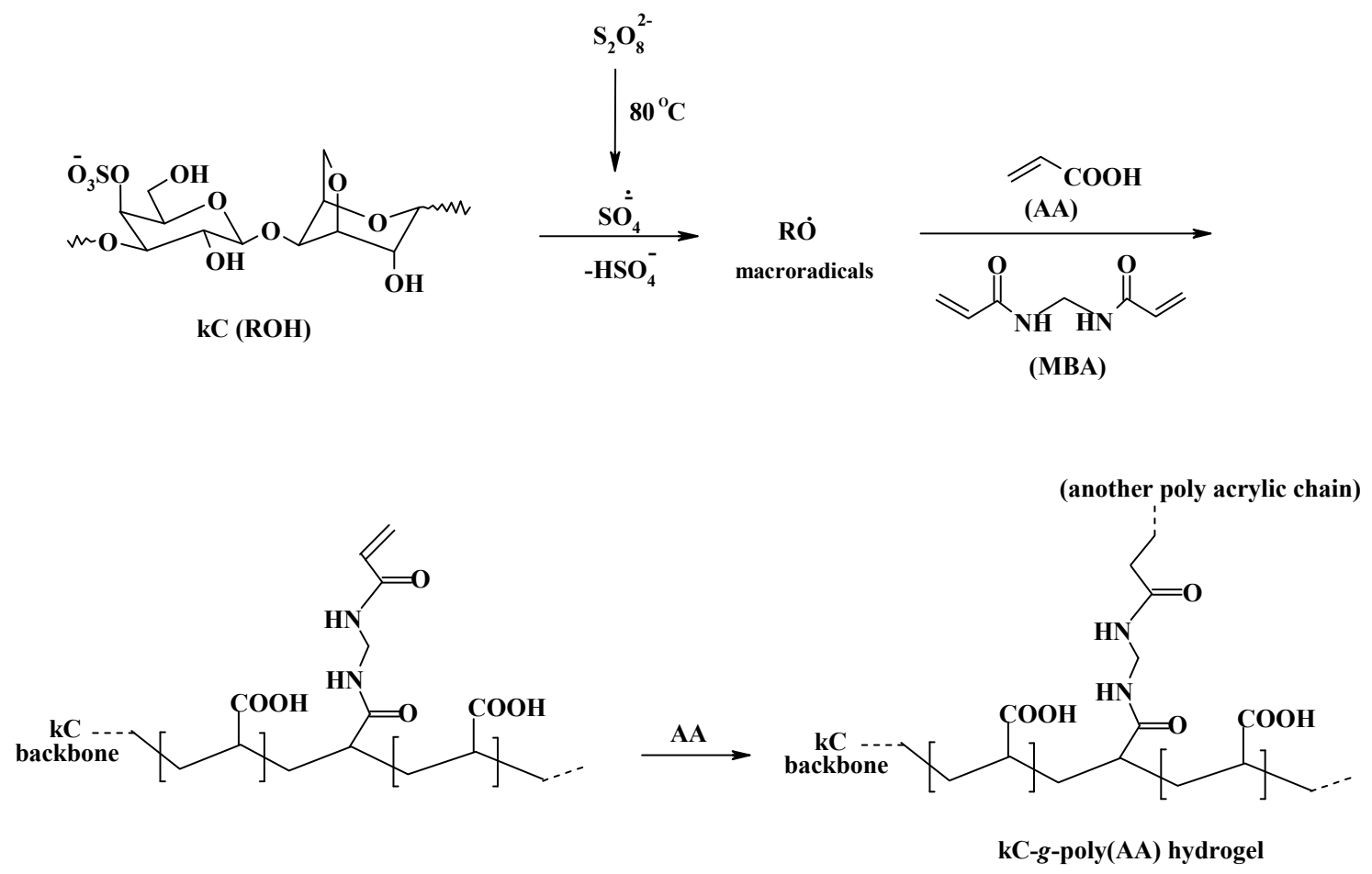

Scheme 1. A proposed mechanism for synthesis of $\kappa \mathrm{C}$-based superabsorbent hydrogel.

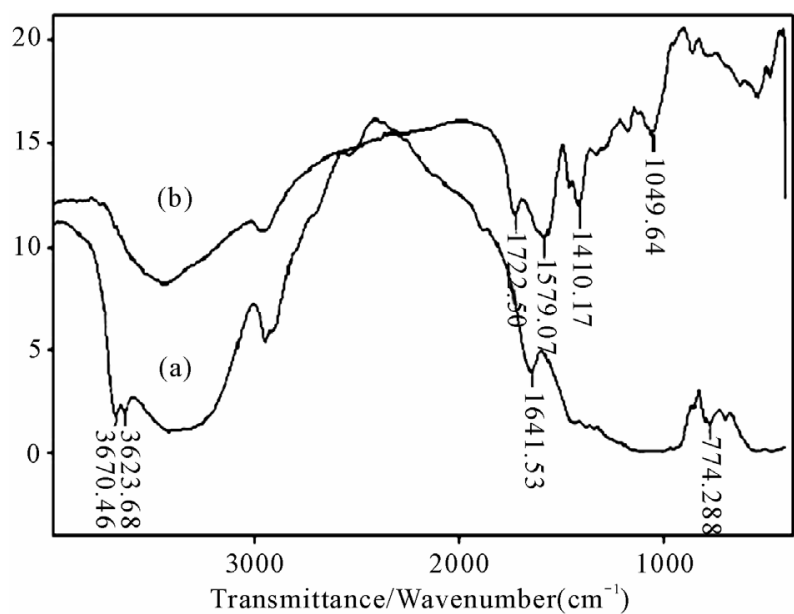

Figure 2. FTIR spectra of the physical mixture of $\kappa \mathrm{C}$ and bentonite (a) and $\kappa \mathrm{C}-\mathrm{g}$-PAANa/bentonite composite.

ponding to the ester groups that can be formed during the graft polymerization reaction. The carboxylate groups of the grafted poly(acrylic acid) can be react with the $-\mathrm{OH}$ groups on the bentonite surface results in the ester formation. The reaction can be shown as follows:

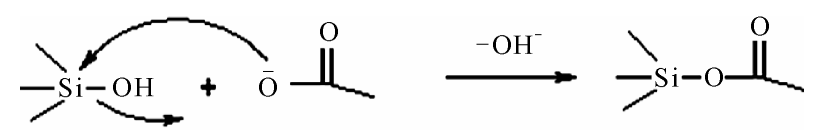

Surface of bentonite Carboxylate anions

\subsection{Scanning Electron Microscopy}

One of the most important properties that must be considered is hydrogel microstructure morphologies. Figure 3 shows the scanning electron microscope images of the hydrogel. This picture verifies that the synthesized polymer have a porous structure. It is supposed that these pores are the regions of water permeation and interaction sites of external stimuli with the hydrophilic groups of the graft copolymers. In this paper, the pores were simply produced from water evaporation during hydrogel synthesis.

The results of BET analysis showed that the average pore diameter of the synthesized hydrogel was $11.5 \mathrm{~nm}$. In general, the size of the pores can be controlled by adjusting the various factors such as the type and amount of surfactant, porosigens and gas forming agent during crosslinking polymerization, and the amount of diluent in the monomer mixture (i.e., monomer-diluent ratio).

\subsection{Effect of MBA Concentration on Swelling}

The swelling ratio as a function of MBA concentration, for crosslinked $\kappa \mathrm{C}$-g-PAA was investigated (Figure 4). Crosslinks is necessary to form a superabsorbent in order to prevent dissolution of the hydrophilic polymer chains in an aqueous environment. As the concentration of MBA was increased, the water absorbency of the superabsorbent composite was decreased. This is due to a 


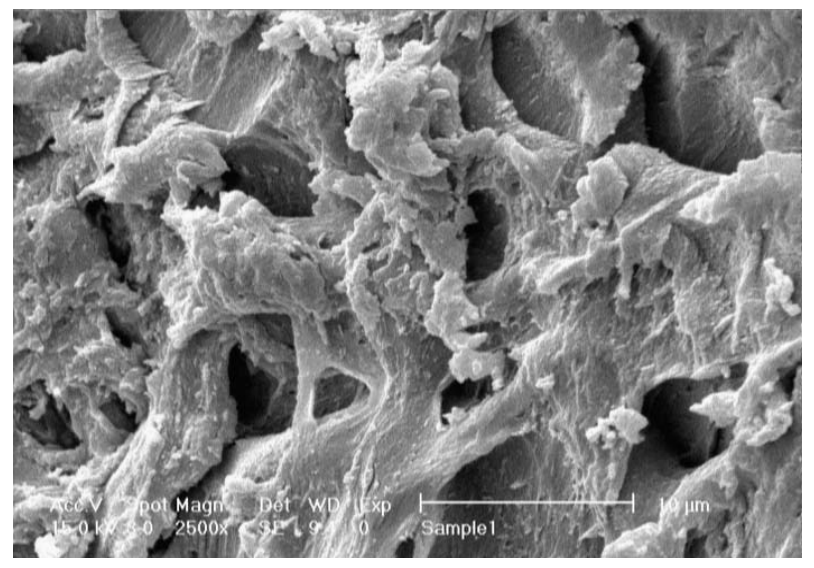

Figure 3. SEM photograph of the hydrogel. Surfaces were taken at a magnification of 2500 , and the scale bar is $10 \mu \mathrm{m}$.

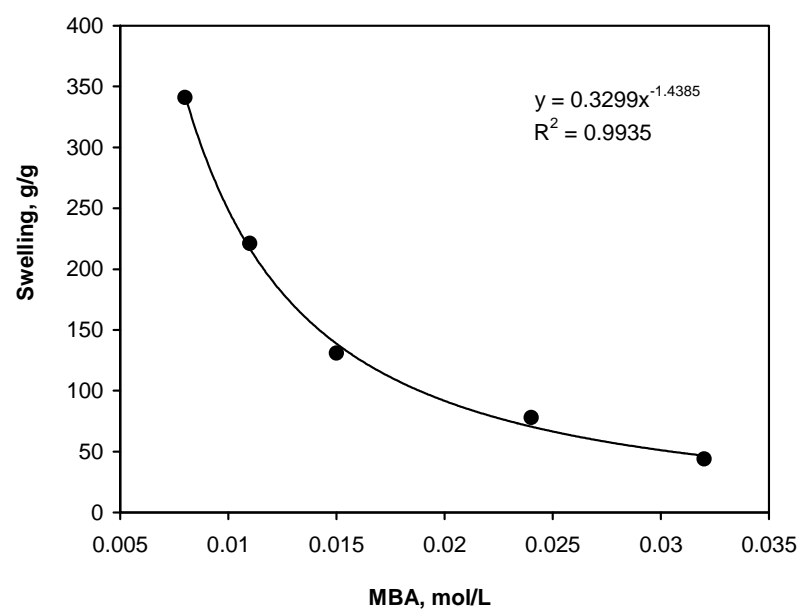

Figure 4. Effect of the crosslinker concentration on water absorbency of the composite.

decrease in the space between the copolymer chains as the crosslinker concentration is increased.

\subsection{Effect of the Monomer Concentration on Swelling}

The effect of the monomer concentration on swelling capacity of $\kappa \mathrm{C}-\mathrm{g}$-PAA composite was investigated (Figure 5). The absorbency is increased versus increasing the AA concentration from 0.50 to $1.80 \mathrm{~mol} / \mathrm{L}$ and then, it is decreased with a further increase for AA. The initial increase in swelling values can be attributed to the higher the hydrophilicity of the hydrogel and the greater availability of AA molecules near the $\kappa \mathrm{C}$ macroradicals. The swelling-loss after the maximum may be attributed to a) preferential homopolymerization over graft copolymerization, b) increase in viscosity of the medium, which restricts the movement of free radicals and monomer molecules, and c) the enhanced chance of chain transfer to monomer molecules.

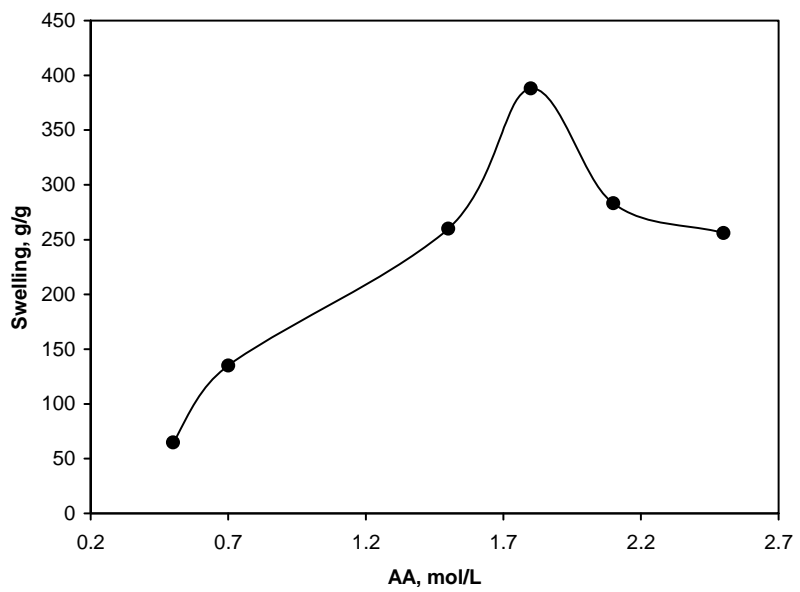

Figure 5. Swelling dependency of the composite on the monomer concentration.

\subsection{Effect of APS Concentration on Swelling}

The effect of initiator content on swelling capacity of crosslinked $\kappa \mathrm{C}$-g-PAA was studied by varying the APS concentration from 0.006 to $0.052 \mathrm{~mol} / \mathrm{L}$ (Figure 6). As shown in the figure, swelling capacity is increased with increasing the APS concentration from 0.006 to 0.023 $\mathrm{mol} / \mathrm{L}$ and then it is considerably decreased with a further increase in the concentration of APS. By increasing the APS concentration up to $0.023 \mathrm{~mol} / \mathrm{L}$, the number of active free radicals on the $\mathrm{\kappa C}$ backbone is increased which, in turn, resulting in higher graft polymerization extent and consequently higher final water absorbency. The APS concentrations higher than the optimum value, however, lead to low-swelling superabsorbents. This swelling-loss may be attributed to an increase in terminating step reaction via bimolecular collision, which, in turn, causes to enhance crosslinking density. Chen and Zhao [31] refer to this possible phenomenon as "selfcrosslinking". In addition, decrease in molecular weight (MW) of grafted PAA of the hydrogel causes to decrease swelling value. The latter reason is due to the inverse relationship between MW and initiator concentration [32]. Moreover, the free radical degradation of $\kappa \mathrm{C}$ backbones by sulfate radical-anions is an additional reason for swelling-loss at higher APS concentration. Hsu et al. report a similar observation in the case of degradation of chitosan with potassium persulfate [33].

\subsection{Effect of Bentonite Amount on Swelling}

The swelling capacity of the superabsorbent composite as a function of bentonite content is illustrated in Figure 7. The bentonite/ $/ \mathrm{C}$ weight ratio was varied from 0.4 to 2.5 , while other reaction variables were constant. The effect of bentonite amount on water absorbency is similar to MBA influence on absorbency. The clay in the polym- 


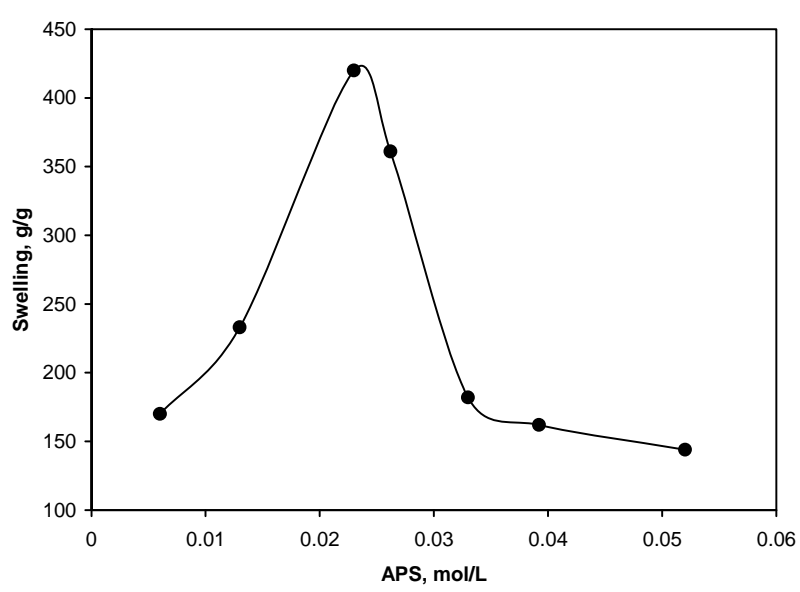

Figure 6. Effect of initiator concentration on water absorbency of the composite.

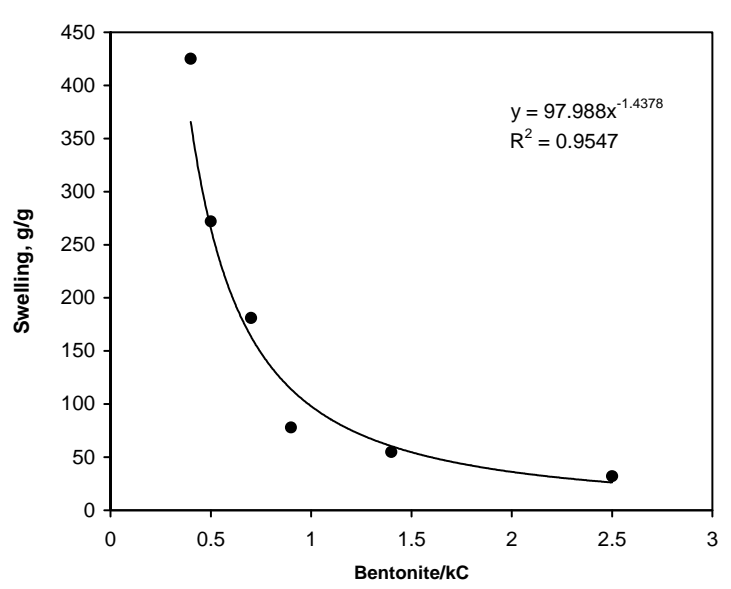

Figure 7. Effect of bentonite/ $\mathrm{C}$ weight ratio on swelling of the hydrogel composite.

erization reaction may be acts via two ways: a) bentonite particles acts as a crosslinking agent (it means that carboxylate groups of sodium poly(acrylate) chains react with bentonite and b) bentonite particles prevent the growing polymer chains through a chain transfer mechanism.

\subsection{Equilibrium Swelling at Various pH Solutions}

Ionic superabsorbent hydrogels exhibit swelling changes at a wide range of $\mathrm{pHs}$. Therefore, in this series of experiments, equilibrium swelling for the synthesized hydrogels was measured in different $\mathrm{pH}$ solutions ranged from 1.0 to 13.0 (Figure 8). Since the swelling capacity of all "anionic" hydrogels is appreciably decreased by addition of counter ions (cations) to the swelling medium, no buffer solutions were used. Therefore, stock $\mathrm{NaOH}$ (pH 13.0) and $\mathrm{HCl}$ (1.0) solutions were diluted with distilled water to reach desired basic and acidic $\mathrm{pHs}$, respect-

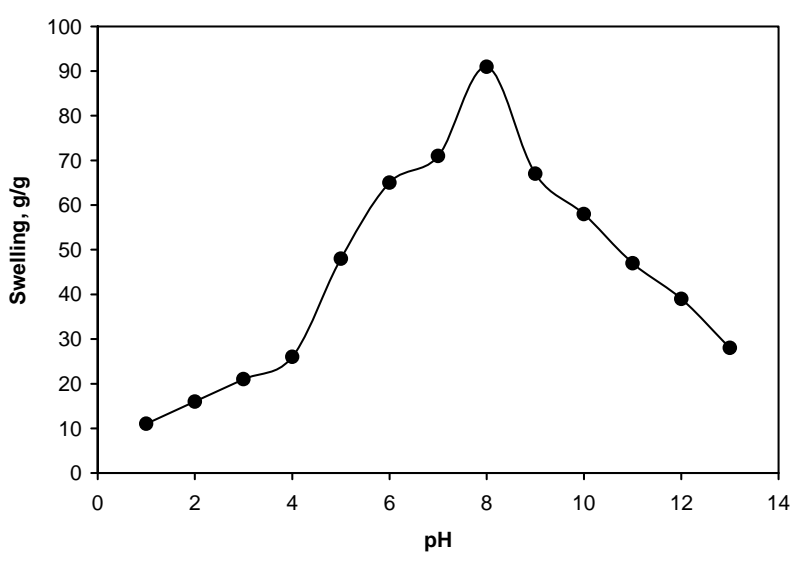

Figure 8. pH-dependent swelling of the superabsorbent composite.

tively. Maximum swelling (91 g/g) was obtained at $\mathrm{pH} 8$. Under acidic pHs $(\leq 4)$, most of the carboxylate anions are protonated, so the main anion-anion repulsive forces are eliminated and consequently swelling values are decreased. However, some sort of attractive interactions (H-O hydrogen bonding) lead to decreased absorbencies. At higher pHs $(5-8)$, some of carboxylate groups are ionized and the electrostatic repulsion between $\mathrm{COO}^{-}$ groups causes an enhancement of the swelling capacity. The reason of the swelling-loss for the highly basic solutions $(\mathrm{pH}>8)$ is "charge screening effect" of excess $\mathrm{Na}^{+}$ in the swelling media, which shields the carboxylate anions and prevents effective anion-anion repulsion.

\subsection{Swelling in Various Salt Solutions}

Swelling capacity in salt solutions is of prime significance in many practical applications such as personal hygiene products and water release systems in agriculture. The swelling ability of "anionic" hydrogels in various salt solutions is appreciably decreased compared to the swelling values in distilled water. This well-known undesired swelling loss is often attributed to a "charge screening effect" of the additional cations which causing a non-perfect anion-anion electrostatic repulsion [34]. Also, in salt solution the osmotic pressure resulting from the difference in the mobile ion concentration between gel and the aqueous phases is decreased and consequently the absorbency amounts are diminished. In the present study, swelling capacity was studied in various chloride salt solutions (Figure 9). As shown in the Figure 9, multivalent cations decrease the swelling capacity considerably. This dramatic decrease of water absorbency in multivalent cationic solutions could be related to the complexing ability of the carboxylate groups inducing the formation of intramolecular and intermolecular complexes, which resulted in an increase in the cross- 


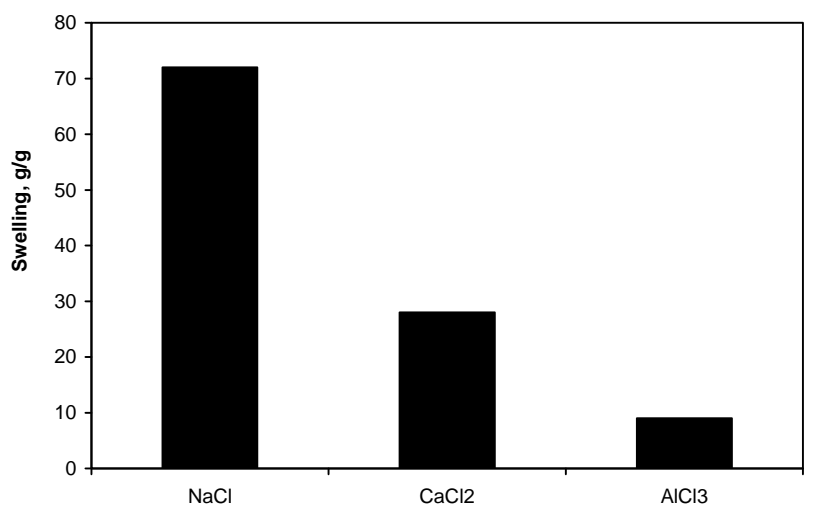

Figure 9. Swelling capacity of the hydrogel in different chloride salt solutions $(0.15 \mathrm{M})$.

linking density of the network [35].

\section{Conclusions}

In the present study, a novel superabsorbent composite based on $\kappa \mathrm{C}$ was prepared by graft copolymerization of AA in the presence of a crosslinking agent. The resultant superabsorbent composite had a large degree of water absorbency. The study of FTIR spectra shows that in the composite spectrum a new absorption band at $1722 \mathrm{~cm}^{-1}$ was appeared that attributed to the ester formation from replacement of hydroxyl groups of bentonite with grafted carboxylate anions onto polysaccharide backbones. The effect of the bentonite amount and MBA concentration showed that with increasing of these parameters, the water absorbency of the superabsorbent composite are decreased.

The swelling of hydrogel exhibited high sensitivity to $\mathrm{pH}$. Study of effect of $\mathrm{H}^{+} / \mathrm{OH}^{-}$concentration carried out at various $\mathrm{pHs}$ shows that the swelling of hydrogel causes several large volume changes. So, we investigated the $\mathrm{pH}$-sensitivity of the hydrogel. Ionic repulsion between charge groups incorporated in the gel matrix by an external $\mathrm{pH}$ modulation could be assumed as the main driving force responsible for such abrupt swelling changes. This superabsorbent network intelligently responding to $\mathrm{pH}$ may be considered as an excellent candidate to design novel drug delivery systems.

Also swelling measurement of the synthesized composites in different salt solutions showed appreciable swelling capacity, especially in $\mathrm{NaCl}$ solution. This behavior is may be due to anti-salt characteristics of the carrageenan part sulfate groups of the superabsorbing networks.

Overall, we report a crosslinking polymerization to achieve superabsorbing composite materials with lower cost and lower salt-sensitivity. The hydrogel composites will most probably posses higher biodegradability (due to the $\kappa \mathrm{C}$ part) and higher swollen gel strength (due to the inorganic parts). The latter properties are of the subjects under consideration in our laboratory.

\section{REFERENCES}

[1] O. V. Khutoryanskaya, Z. A. Mayeva, G. A. Mun and V. V. Khutoryanskiy, "Designing Temperature-Responsive Biocompatible Copolymers and Hydrogels Based on 2Hydroxyethyl(meth)acrylates," Biomacromolecules, Vol. 9, No. 12, 2008, pp. 3353-3361. doi:10.1021/bm8006242

[2] W. E. Hennink and C. F. van Nostrum, "Novel Crosslinking Methods to Design Hydrogels," Advanced Drug Delivery Reviews, Vol. 54, No. 1, 2002, pp. 13-36. doi:10.1016/S0169-409X(01)00240-X

[3] F. L. Buchholz and A. T. Graham, "Modern Superabsorbent Polymer Technology,” Wiley, New York, 1997.

[4] L. B. Peppas and R. S. Harland, "Absorbent Polymer Technology,” Elsevier, Amsterdam, 1990.

[5] R. Po, "Water-Absorbent Polymers: A Patent Survey," Journal of Macromolecular Science-Reviews in Macromolecular, Vol. 34, No. 4, 1994, pp. 607-662.

[6] J. Kost, "Encyclopedia of Controlled Drug Delivery,” E. Mathiowitz, Ed., Wiley, New York, 1999, p. 445.

[7] N. A. Peppas and A. G. Mikes, "Hydrogels in Medicine and Pharmacy," CRC Press, Boca Raton, Vol. 1, 1986.

[8] J. P. Fisher, S. Jo, A. G. Mikos and A. H. Reddi, “Thermoreversible Hydrogel Scaffolds for Articular Cartilage Engineering," Journal of Biomedical Materials Research, Vol. 71A, No. 2, 2004, pp. 268-274. doi:10.1002/jbm.a.30148

[9] S. J. Bryant, K. L. Durnad and K. S. Anseth, "Manipulations in Hydrogel Chemistry Control Photoencapsulated Chondrocyte Behavior and Their Extracellular Matrix Production," Journal of Biomedical Materials Research, Vol. 67A, No. 4, 2003, pp. 1430-1438. doi:10.1002/jbm.a.20003

[10] H. Omidian, J. G. Rocca and K. Park, "Advances in Superporous Hydrogels," Journal of Controlled Release, Vol. 102, No. 2, 2005, pp. 3-12. doi:10.1016/i.jconrel.2004.09.028

[11] S. J. Smit, "Superabsorbent Polymer Having Improved Absorption Rate and Absorption Under Pressure," US Patent 5399591, 1995.

[12] R. Chambers, "Superabsorbent Polymers and Products Therefrom," US Patent 5597873, 1997.

[13] A. Mitchell, "Method of Manufacturing a Semiconductor Device," US Patent 6376318, 2002.

[14] K. Kabiri and M. J. Zohuriaan-Mehr, "Superabsorbent Hydrogel Composites," Polymers for Advanced Technologies, Vol. 14, No. 6, 2003, pp. 438-444. doi:10.1002/pat.356

[15] J. Lin, J. Wu, Z. Yang and M. Pu, "Synthesis and Properties of Poly(acrylic acid)/Mica Superabsorbent Nanocomposite," Macromolecular Rapid Communications, Vol. 
22, No. 6, 2001, pp. 422-424. doi:10.1002/1521-3927(20010301)22:6<422::AID-MAR C422>3.0.CO;2-R

[16] J. Wu, J. Lin, G. Lin and C. Wei, "Influence of the $\mathrm{COOH}$ and COONa Groups and Crosslink Density of Poly(Acrylic Acid)/Montmorillonite Superabsorbent Composite on Water Absorbency," Polymer International, Vol. 50, No. 9, 2001, pp. 1050-1053. doi:10.1002/pi.728

[17] K. Haraguchi, T. Takenisa and S, Fan S, "Effects of Clay Content on the Properties of Nanocomposite Hydrogels Composed of Poly(N-isopropylacrylamide) and Clay," Macromolecules, Vol. 35, No. 27, 2002, pp. 10162-10171. doi:10.1021/ma021301r

[18] K. Haraguchi and T. Takenisa, "Nanocomposite Hydrogels: A Unique Organic-Inorganic Network Structure with Extraordinary Mechanical, Optical, and Swelling/ Deswelling Properties," Advanced Materials, Vol. 14, No. 16, 2002, pp. 1120-1128.

doi:10.1002/1521-4095(20020816)14:16<1120::AID-AD MA1120>3.0.CO;2-9

[19] Y. Liu, M. Zhu, X. Liu, W. Zhang, B. Sun and Y. Chen, "Synthesis and Characterization of Novel Monomers and Polymers Containing Chiral (-)-Menthyl Groups," Polymer, Vol. 47, No. 14, 2006, pp. 4901-4908. doi:10.1016/i.polymer.2006.05.043

[20] K. Xu, J. Wang, S. Xiang, Q. Chen, Y. Yue and X. Su, "Poly-Ampholytes Superabsorbent Nanocomposites with Excellent Gel Strength," Composites Science and Technology, Vol. 67, No. 15, 2007, pp. 3480-3486. doi:10.1016/j.compscitech.2007.02.009

[21] V. D. Athawale and V. Lele, "Graft Copolymerization Onto Starch. 3: Grafting of Acrylamide Using Ceric Ion Initiation and Preparation of Its Hydrogels," Starch/ Starke, Vol. 50, No. 10, 1998, pp. 426-432. doi:10.1002/(SICI)1521-379X(199810)50:10<426::AIDSTAR426>3.0.CO;2-\#

[22] D. W. Lim, H. S. Whang and K. J. Yoon, "Synthesis and Absorbency of a Superabsorbent from Sodium Starch Sulfate-g-Polyacrylonitrile," Journal of Applied Polymer Science, Vol. 79, No. 8, 2003, pp. 1423-1430. doi:10.1002/1097-4628(20010222)79:8<1423::AID-APP 90>3.0.CO;2-V

[23] Y. Sugahara and O. Takahisa, "Synthesis of Starch-GraftPolyacrylonitrile Hydrolyzate and Its Characterization," Journal of Applied Polymer Science, Vol. 82, No. 6, pp. 1437-1443. doi:10.1002/app.1981

[24] J. M. Joshi and S.V. Kumar, "Graft Copolymerization of 2-Hydroxyethylmethacrylate onto Carboxymethyl Chitosan Using CAN as an Initiator," Polymer, Vol. 47, No. 6, 2006, pp. 2198-2204. doi:10.1016/j.polymer.2005.11.050

[25] J. Lin, J. Wu, Z. Yang and M. Pu, "Synthesis and Proper- ties of Poly(Acrylic Acid)/Montmorilonite Superabsorbent Composites," Polymers and Polymer Composites, Vol. 9, No. 7, 2001, pp. 469-471.

[26] J. Wu, J. Lin, M. Zhou and C. Wei, "Synthesis and Properties of Starch-Graft-Polyacrylamide/Clay Superabsorbent Composite," Macromolecular Rapid Communications, Vol. 21, No. 15, 2000, pp. 1032-1034. doi:10.1002/1521-3927(20001001)21:15<1032::AID-MA RC1032>3.0.CO;2-N

[27] J. Wu, Y. Wei, J. Lin and S. Lin, "Study on Starch-gAcrylamide/Mineral Powder Superabsorbent Composite," Polymer, Vol. 44, No. 21, 2003, pp. 6513- 6520. doi:10.1016/S0032-3861(03)00728-6

[28] R. E. Kirk and D. F. Othmer, "Encyclopedia of Chemical Technology," 4th Edition, John Wiley \& Sons, New York, 1992, Vol. 4, p. 942.

[29] A. Pourjavadi and M. J. Zohuriaan-Mehr, "Modification of Carbohydrate Polymers via Grafting in Air. 1. Ceric-Induced Synthesis of Starch-g-Polyacrylonitrile in Presence and Absence of Oxygen," Starch/Starke, Vol. 54, No. 3-4, 2002, pp. 140-147. doi:10.1002/1521-379X(200204)54:3/4<140::AID-STAR $140>3.0 . \mathrm{CO} ; 2-\mathrm{I}$

[30] A. Pourjavadi and M. J. Zohuriaan-Mehr, "Modification of Carbohydrate Polymers via Grafting in Air. 2. CericInitiated Graft Copolymerization of Acrylonitrile onto Natural and Modified Polysaccharides," Starch/ Starke, Vol. 54, No. 10, 2002, pp. 482-488. doi:10.1002/1521-379X(200210)54:10<482::AID-STAR $\underline{482>3.0 . \mathrm{CO} ; 2-\mathrm{I}}$

[31] J. Chen and Y. Zhao, "Relationship between Water Absorbency and Reaction Conditions in Aqueous Solution Polymerization of Polyacrylate Superabsorbents," Journal of Applied Polymer Science, Vol. 75, No. 6, 2000, pp. 808-814.

doi:10.1002/(SICI)1097-4628(20000207)75:6<808::AIDAPP10>3.0.CO;2-3

[32] J. Branrup and E. H. Immergut, "Polymer Handbook," 3rd Edition, New York, Wiley, 1989.

[33] S. C. Hsu, T. M. Don and W. Y. Chiu, "Free Radical Degradation of Chitosan with Potassium Persulfate," Polymer Degradation and Stability, Vol. 75, No. 1, 2002, pp. 73-83. doi:10.1016/S0141-3910(01)00205-1

[34] P. J. Flory, "Principles of Polymer Chemistry," Cornell University Press, New York, Ithaca, 1953.

[35] D. Castal, A. Ricard and R. Audebert, "Swelling of Anionic and Cationic Starch-Based Superabsorbents In Water and Saline Solution," Journal of Applied Polymer Science, Vol. 39, No. 1, 1990, pp. 11-29. doi:10.1002/app.1990.070390102 